\title{
Aeolian Artefacts
}

\author{
Juan Carlos Duarte Regino \\ Aalto University \\ Helsinki, Finland \\ juan.duarte@aalto.fi
}

\section{ABSTRACT}

Amid the history of sound instruments, it's possible to find an ancient drift in service of expanding human sensory to increase the understanding of natural forces, including structures and devices that took advantage of natural forces, such as wind current and ocean waves: these environmental agents represented entities with own will that informed about natural cycles or premonitory capabilities. Comparably, in our present times, networks of hybrid computer sensing systems could be used to provide an expanded knowledge about climate transformations on a planetary scale.

The present project consists of a sonic artefact, designed to create an Augmented Listening experience based on wind forces and radio signal transmission: where any change in an outdoor environment is used to drive a generative live sonification that is transmitted back on real time over FM radio to the outdoor environment. The work proposes an speculative approach on potential correlations between wind forces and electromagnetic transmissions, and a notional interspecies communication channel.

\section{KEYWORDS:}

Augmented Listening, Generative Soundscape, Aeolian.

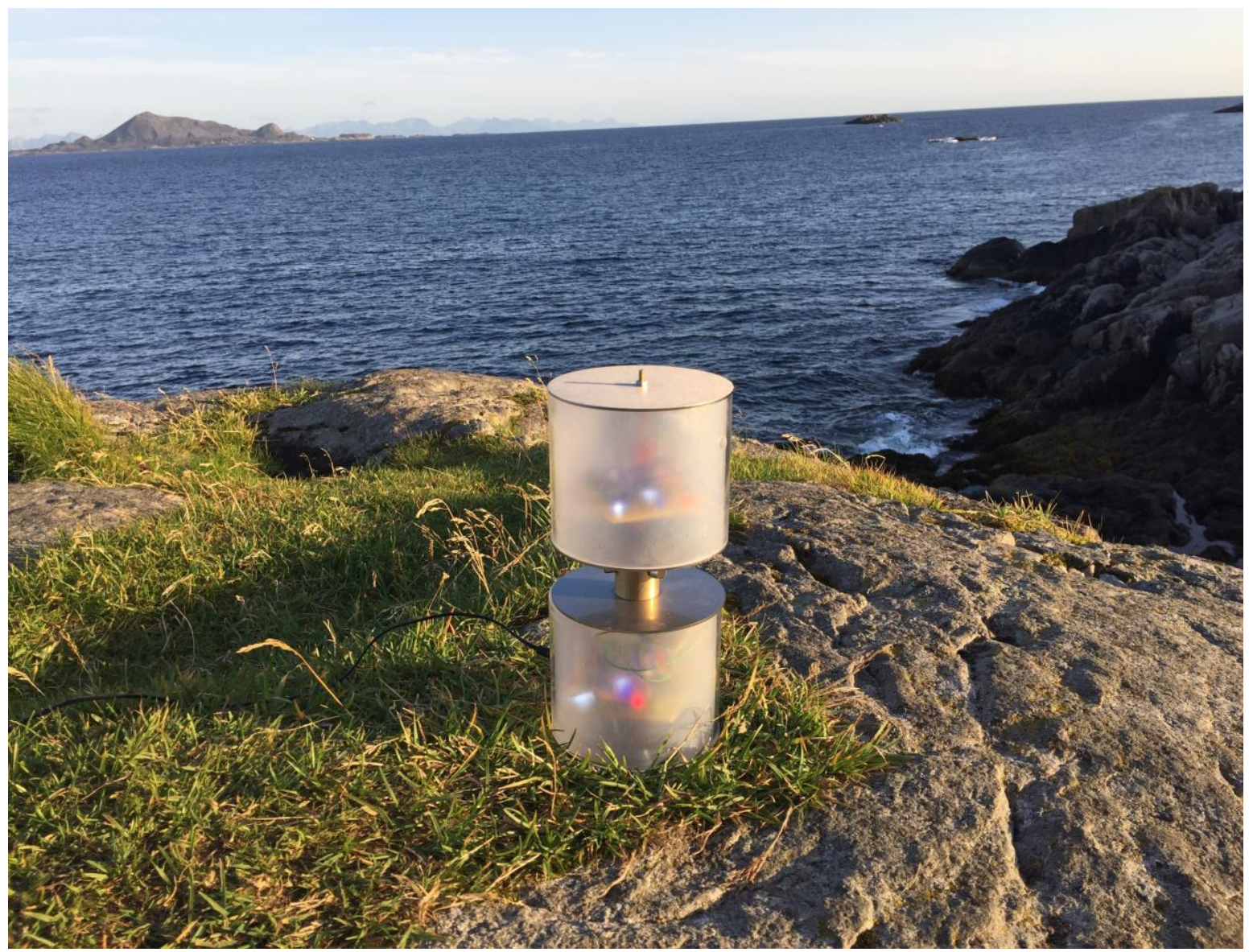

Fig.1 Picture from a Sensing Node of Aeolian Artefacts, used for field recordings. 


\section{INTRODUCTION}

As part of an artistic practiced based research on Environmental Media, Aeolian Artefacts is interested in designing tools for artistic production, blending generative media arts with the study of environment, in order to speculate on potential correlations between media ecologies and environments.

Aeolian Artefacts attempts to make audible changes in wind (force and direction). When these are detected, the electro-acoustic impulses are processed on real time with a portable computing system that can be easily placed in remote locations, to ease field recordings and radio transmission in situ. Thus, the sonic artefacts comprise a set of sensing-transmission nodes that are used to create a generative sound piece that mediates information from an ecosystem of environmental and electromagnetic signals. The different dynamics of the wind are captured as data to be used as sonic materials to describe a phenomenon that encompasses the micro to the macro temporal structure. The resulting sound piece emphasizes the wind as an event disseminated and reconstructed employing sound synthesis and radio signals, articulated as a system of functions, resembling the cycles of climate on our planet.

\section{THE AEOLIAN IN SONIC ARTS}

The main reference to the present project is an ancient instrument known as the Aeolian Harp (cf. Kircher, A. ca 1650.), that was used as an object tp enabled sacred spaces, such as mountains and temples, to "have a voice" according to a automatic mechanism of strings and resonators, that was played solely by the aleatoric forces of wind. This artistic practice achieved the concretion of pioneer scientific advances in acoustics, music theory, and instrument invention to register and understand the cycles of the aeolian phenomena. Recently, sound artists and experimental musicians, including Gordon Monahan and John Grzinich, have worked on novel implementations of the Aeolian Harp, using the same principle as Kircher, but improving on custom made variations to record ambient interactions of wind with string to-building resonators that activate sonically spaces, and produce uncanny sound textures that blend wind forces with material and spatial qualities into a multi layered sonic generative installations.

In this sense, Timothy Morton (2009) describes the Aeolian as an element of Ambient Poetics, where the creation of sound is disembodied, or emanating from an unseen source. The sound produced is coming from a diffuse medium that involves the medium: as in this case, the wind and spatial configurations work together as an ensemble.
Besides, Morton underlines that this practice is often present in Experimental music when sounds play through speakers, as Acousmatic sources, that obscure the source of sound (either prerecorded materials or synthetic produced live sound). Along with this line, Morton employs the term Eco-mimesis as a register that is created in environmental art, to makes us aware of the atmosphere. Thus, the Aeolian is generated "from the background" (wind forces interacting with spatial relations) but appears "in the foreground" as an Acousmatic focal source of a sound. This intrinsic quality of the Aeolian brings listening as a relational activity with environing properties.

\section{AUTO-POEIESIS}

Takin into account that the notion of Aeolian provides a framework for automatic artistic creation (given that is independent of the author's intention), that is suitable to the idea mentioned above of Ambient Poetics, based on interactions between an artifact and environmental conditions. It is worth to consider here the concept of Autopoiesis of Maturana and Varela (1972), that serves as a starting point towards the design of systems that are by themselves nests, maintained and reproducible. In this sense, Enactivism is a central proposal on how species fit into the world: that is, autopoiesis as a design model indicates the need for entities to receive and modify their environment in an ecosystem of agents that not only processes information from passive way but react and adapt to the conditions offered by the environment. More recently, the idea of auto-poetic systems is present in the field of computer systems, precisely in generative art. By combining information and processing systems, that implies mutual, such as organic behaviours is comparable to a natural ecosystem (for example, the relationships established between the organisms that inhabit a forest, including food chains, natural resources, and climate cycles through the seasons of the year).

\section{ENVIRONMENTAL COMPUTING}

Jennifer Gabrys (2016) enounces that the idea of programming (sensing) of the planet is as a process anchored since the space age when the use of satellites orbiting around the world was used to retrieve information, data and produce image registration nodes. The system would allow a reprogramming of the planet according to a view from outside the earth. In this context, the idea of environmental computing is interested in how environments generated content from the operation of sensors and the forms of programming as a source of unpredictable results. Beyond a speculative perspective, Jennifer Gabrys's project Citizen Sensing focuses on proposing structural models on environmental monitoring seen as a complex system that varies according to 
environmental conditions. Based on this information, Citizen Sensing works on projects to create civil tools specialized in dealing with various types of environmental effects, including, flora and fauna transformations, or the study of climate change.

The current technological juncture of ubiquitous computing offers accessible and open tools to program sensor systems, which react to processes related to networks of nodes, dispersed in the geographical space and to temporalities around climate cycles and natural resources such as water and wind. The purpose of Citizen Sensing beyond the accumulation of sensory data and is about informing about the experience in an environment and how this experience allows the creation of artifacts that react collaboratively with other artificial and natural species.

Moreover, the work of pioneer media artist, Roy Ascott (1990) pointed out the importance of Telematic Art (of remote participation) as a way to enable thinking of planetary relations as a whole. By using satellite dispersed geographically, the artists could benefit from using remote sensing devices, which connected human mind with artificial systems of intelligence and perception. This idea inherited the vision of wholeness from the Gesamtkunstwerk, including data work from intellect, emotions, and sensibility of the observer to amplify the mind into unpredictable configurations of thought and creativity. Such ideas, according to Ascott, have been pointed out by Gregory Bateson (1972) when described the concept of "mind-at-large" to facilitate the study of Ecologies.

\section{FINAL REMARKS}

As a study of Augmented Listening, the installation conceives a reflection on micro and macrostructural transformations of the wind dynamics, to produce a database of patterns generated over long periods of time. This analysis could be used to create a map of wind fluxes that stretches from the immediate perceivable to the long-lasting wind transformations. The importance of arranging different lengths of time in wind events is correlated to a resulting, ever transforming, sound composition that echoes past, current and possible states of transitions.

Such analysis of time patterns from wind flux relies on the network of sensor nodes, to provide a summary of multiple data streams that feed a neural network program, which role is to create patterns that combine wind soundscapes, radio signals, and data sonification as sonic elements to play with the sonic artefact.

The research led by Aeolian Artefacts aims to explore the potential of tools to analyse and produce a generative artwork from the environment that reflects on potential correlations between aelian forces and data to sound radio transmission. The design process of this artefact works as an assembly of artificial intelligence that serves to make significant and perceptible their surrounding interactions by augmenting the listening perception. The experience produced by the artwork aims to involve participants in an enhanced listening, boosted by pervasive technology to create a novel mapping of environing events into human perceivable signals.

The project is being documented with recordings from exhibitions and field recordings at the following webpage: https://juanduarteregino.com/Artefactos-Eolicos

\section{REFERENCES}

Morton, Timothy (2009). Ecology without Nature. Rethinking Enviornmental Aesthetics. Harvard University Press.

Gabrys, Jeniffer (2016). Program Earth. Environmental Sensing Technology and the Making of a Computational Planet. University of Minessota Press.

Ascott, Roy (1990) Is there Love in the Telematic Embrace? Art Journal, Vol. 49, No. 3, Computers and Art: Issues of Content (Autumn, 1990), pp. 241-247

Bateson, Gregory. Steps to an Ecology of Mind. (1972). Collected Essays in Anthropology, Psychiatry, Evolution, and Epistemology. University of Chicago Press.

Maturana, Humberto R. Varela, Francisco. (1972). Autopoiesis and Cognition. The realization of the living. D. Reidel Publishing Company. Dordrecht: Holland / Boston. 